\title{
Functional Data Analysis of Spectroscopic Data with Application to Classification of Colon Polyps
}

\author{
Ying Zhu \\ Mathematics and Mathematics Education, National Institute of Education, Nanyang Technological University, Singapore City, \\ Singapore \\ Email: ying.zhu@nie.edu.sg
}

How to cite this paper: Zhu, Y. (2017) Functional Data Analysis of Spectroscopic Data with Application to Classification of Colon Polyps. American Journal of Analytical Chemistry, 8, 294-305.

https://doi.org/10.4236/ajac.2017.84022

Received: March 3, 2017

Accepted: April 27, 2017

Published: April 30, 2017

Copyright $\odot 2017$ by author and Scientific Research Publishing Inc. This work is licensed under the Creative Commons Attribution-NonCommercial International License (CC BY-NC 4.0).

http://creativecommons.org/licenses/by-nc/4.0/

\begin{abstract}
In this study, two functional logistic regression models with functional principal component basis (FPCA) and functional partial least squares basis (FPLS) have been developed to distinguish precancerous adenomatous polyps from hyperplastic polyps for the purpose of classification and interpretation. The classification performances of the two functional models have been compared with two widely used multivariate methods, principal component discriminant analysis (PCDA) and partial least squares discriminant analysis (PLSDA). The results indicated that classification abilities of FPCA and FPLS models outperformed those of the PCDA and PLSDA models by using a small number of functional basis components. With substantial reduction in model complexity and improvement of classification accuracy, it is particularly helpful for interpretation of the complex spectral features related to precancerous colon polyps.
\end{abstract}

\section{Keywords}

Functional Principal Component Analysis, Functional Partial Least Squares, Functional Logistic Regression, Principal Component Discriminant Analysis, Partial Least Squares Discriminant Analysis

\section{Introduction}

Near-infrared (NIR) spectra of biomedical objects consist of many overlapping absorption bands representing the different modes of vibration of a large number of molecular constituents in the compounds, which are sensitive to the physical and chemical states of the compounds [1] [2] Spectroscopic data show highly correlated structure due to the complex nature of its spectral absorption bands. The underlying information thus varies smoothly with spectral wave- 
length. This feature makes spectral data different from the typical statistical high-dimensional data.

Traditional spectral data analysis involves multivariate statistical techniques such as multiple linear regression, principal components regression (PCR) [3] [4] and partial least squares (PLS) regression [5] [6]. However, these methods consider the spectrum as a set of discrete variable points rather than as a continuous function. From a physical point of view it would be more informative to describe the spectrum as a smooth function, being a sum of absorption peaks caused by the different chemical constituents present in the sample under study, where the absorbance at two wavelengths close to each other is shown highly correlated.

Functional data analysis (FDA) proposed by Ramsay and Silverman [7] is used to deal with data of a functional nature, where each spectrum is replaced by its approximation by a linear combination of smooth basis functions. In recent years, there have been an increasing number of publications on FDA, including functional linear regression with functional predictors and scalar responses, and its applications in NIR spectroscopy, particularly in the area of biomedical applications [8] [9] [10]. In classification problems, generalized linear model was adapted to the presence of functional predictor variables [11] [12]. To deal with the problem of multicollinearity in the multiple linear regression of functional data, functional principal component analysis and functional partial least squares were introduced to the classification problem of functional data [13].

In this study of functional logistic regression (FLR) [14] models with different functional basis were explored to distinguish the differences between the two polyps in the colon for detection of precancerous adenomatous polyps. PCA basis and PLS basis [7] were investigated and insights were gained on the effects of different functional representations of predictors. The multivariate methods using principal component discriminant analysis (PCDA) and partial least squares discriminant analysis (PLSDA) were also explored for classification. The performances of the FLR models were compared with that of the PCDA and PLSDA models in terms of both classification accuracy and understanding of spectral features.

\section{Materials and Methods}

\subsection{Spectroscopic Data Description}

A colon dataset is presented here to illustrate the application of functional data analysis in a classification setting with a problem arising in colonoscopy. The evolution of colon cancer starts with colon polyps. There are two different types of colon polyps, hyperplastic and adenomatous polyps. Hyperplasias are benign polyps which are known not to evolve into cancer. Adenomas have a strong tendency to develop cancer and therefore they have to be excised immediately. Polyps are often found during endoscopy of the colon (colonoscopy). A method to differentiate reliably adenomas from hyperplasias during a preventive medical colonoscopy is highly desirable. 
Visible-NIR spectra covering the range from 320 to $920 \mathrm{~nm}$ were collected from 64 human colonic biopsy sites using an optical probe in vivo during colonoscopy which was undertaken to assess patients for precancerous adenomatous polyps. Repeated measurements, on average 6 spectra per site, were taken from each biopsy site. In total 363 spectra were analyzed, including 63 spectra from 11 hyperplastic polyps and 300 spectra from 53 adenomatous polyps.

\subsection{Spectral Pre-Treatment}

Before constructing a classification model, standard data pre-processing was carried out on the spectra to improve signal quality. This involved spectral smoothing using Savitzky-Golay method (Savitzky and Golay 1964) [15], cropping the noisy ends of the spectra, and normalizing using the standard normal variate (SNV) method [16]. After pretreatment, only the spectra in the range of $370-800 \mathrm{~nm}$ were used in our analysis. Spectra from the two types of polyps are of very similar overall shape, with the mean spectra showing small between-class differences as shown in Figure 1.

\subsection{Statistical Analysis}

\subsubsection{Principal Component Discriminant Analysis (PCDA) and Partial Least Squares Discriminant Analysis (PLSDA)}

Multivariate statistical methods including principal component analysis (PCA), partial least squares (PLS) and linear discriminant analysis (LDA) [17] [18] [19] were employed to investigate the differences of spectra from hyperplastic and adenomatous polyps. Direct implementation of LDA in high-dimensional spectroscopic data setting provides poor classification results and the interpretation of the results is challenging due to singularity problem and highly-correlated spectral features. To solve this problem, PCA or PLS was used to construct a reduced number of new uncorrelated variable with maximal variance or maximal covariance with response. Then LDA focuses on finding a linear combination of the new variables to construct canonical variate which gives the maximum separation of the two groups. Using pretreated spectral data described in Section 2.2, classification rules were derived using principal component discriminant analysis (PCDA) [20] [21] [22] and partial least squares discriminant analysis (PLSDA) [20] [21] [22] [23]. The PCDA involves an initial PCA on the pre-treated spectra followed by a LDA performed on the first $\mathrm{k}$ PCs' scores. The PLSDA involves a PLS regression on the pre-treated spectral data using as response variable y a dummy variable, coded as 0 or 1 for spectrum from hyperplastic and adenomatous polyps respectively. This data reduction step is followed by a LDA on the first $k$ PLS components' scores. Both PCDA and PLSDA were carried out with $k$ ranging from 2 to 30 and the number of components $k$ was optimized by using cross-validation. The leave-out-one-site cross-validation was used in this work to train the algorithm by carrying out the classification rules on all the data except one biopsy site which was then tested. This was repeated until all sites have been tested and an overall model accuracy was determined. This was used to as- 
sess the performance of the classification as measured by sensitivity and specificity.

\subsubsection{Functional PCA and Functional PLS Regression}

Though multivariate PCA and PLS are powerful exploratory statistical methods to reduce the dimension of the original spectral variable, in spectroscopic data setting it would be more informative to describe the spectrum as a smooth function rather than as a set of points when taking into account the underlying spectral information.

To solve the problems of high dimensionality and multicollinearity encountered in functional regression models, the PCA and PLS have been generalized to the infinite-dimensional case where observations of predictor variables are curves or functions (functional data) instead of vectors as in multivariate case. Functional PCA [7] [13] and Functional PLS [7] [8] [13] [14] are useful tools for studying functional data by providing common functional components so as to analyze the variability of functional data in an understandable manner. By approximating infinite-dimensional random functions by a finite number of random score vectors, functional PCA appears as a dimension reduction technique just as in the multivariate case and thus reduces the complexity of the spectroscopic data.

Let $\left\{x_{1}(t), \cdots, x_{n}(t): t \in T\right\}$ be a random sample of observations (sample paths) of a functional predictor variable $X(t)$ where $x_{i}(t)$ is the functional predictor of $i$ th spectrum with $t$ the function argument. In this study, $T$ is the spectral wavelength interval extending from $370 \mathrm{~nm}$ to $800 \mathrm{~nm}$. Let $\left\{y_{1}, \cdots, y_{n}\right\}$ be a set of observations of a binary response variable $Y$ where each $y_{i}$ is a dummy variable, coded as either 0 or 1 for the $i$ th spectrum from either hyperplastic or adenomatous polyps respectively. We assume that the random variables $X$ and $Y$ are defined on the same probability space with $X$ valued in the space $L_{2}$ of square integrable functions on $T$.

Functional PC and functional PLS components are uncorrelated that are obtained as generalized linear combinations of the functional predictor variables. The $j$ th component is defined by

$$
z_{j}=\int_{T} x(t) w_{j}(t) \mathrm{d} t
$$

where $w(t)$ are weight functions that are the solutions to the following optimization problems.

For functional PCA, we seek weight functions $w(t)$ such that the variance of components defined in Equation (1) is maximal.

$$
\arg \max _{w} \operatorname{Var}\left(\int_{T} x(t) w(t) \mathrm{d} t\right)
$$

subject to $\int_{T} w^{2}(t) \mathrm{d} t=\|w\|^{2}=1 ; \int_{T} w_{k}(t) w_{m}(t) \mathrm{d} t=0, \forall k<m$.

For functional PLS, we seek weight functions $w(t)$ such that the covariance between the response and latent components constructed in Equation (1) is maximal.

$$
\arg \max _{w} \operatorname{Cov}^{2}\left(\int_{T} x(t) w(t) \mathrm{d} t, Y\right) \text { subject to }\|w\|^{2}=1
$$




\subsubsection{Functional Logistic Regression (FLR) with Functional PC Basis and Functional PLS Basis}

In binary classification problems, a functional logistic regression (FLR) model is used to model the relationship between the binary response and the functional predictor whose observations are functions instead of vectors as in multivariate case. The FLR model is given by

$$
y_{i}=\pi_{i}+\varepsilon_{i}, i=1, \cdots, n
$$

where $\pi_{i}$, the probability that the binary response variable takes value one given a functional observation, is modeled as

$$
\pi_{i}=P\left(y_{i}=1 \mid x_{i}(t): t \in T\right)=\frac{\exp \left\{\alpha+\int_{T} x_{i}(t) \beta(t) \mathrm{d} t\right\}}{1+\exp \left\{\alpha+\int_{T} x_{i}(t) \beta(t) \mathrm{d} t\right\}}, i=1, \cdots, n,
$$

with $\alpha$ being a real parameter, $\beta(t)$ the functional parameter that belongs to the space $L_{2}$, and $\varepsilon_{i}$ are independent random errors.

Taking the logit transformation gives the following equivalent expression for FLR model:

$$
l_{i}=\ln \frac{\pi_{i}}{1-\pi_{i}}=\alpha+\int_{T} x_{i}(t) \beta(t) \mathrm{d} t .
$$

The use of least squares criteria to estimate the parameters of the functional linear regression model yields an ill-posed problem due to the infinite dimension of the predictor space. In addition, sample path $x_{i}(t)(i=1, \cdots, n)$ are measured discretely. The most common solution to this problem is to assume that both the sample paths $x_{i}(t)$ and the parameter function $\beta(t)$ belong to a finite dimension space spanned by a basis of functions. We then have following representations of $x_{i}(t)$ and $\beta(t)$ as a linear combination of the basis functions $\phi$ :

$$
x_{i}(t)=a_{i}^{\prime} \phi, \beta(t)=\beta^{\prime} \phi,
$$

where $\phi=\left(\phi_{1}(t), \cdots, \phi_{K}(t)\right)^{\prime}$ is a vector of basis functions, $a_{i}=\left(a_{i 1}, \cdots, a_{i K}\right)^{\prime}$ is the vector of sample path basis coefficients and $\beta=\left(\beta_{1}, \cdots, \beta_{K}\right)^{\prime}$ is the parameter function basis coefficients. Therefore, the functional linear regression model is equivalent to the multiple linear model

$$
L=1 \alpha+\mathrm{A} \Phi \beta,
$$

where $L=\left(l_{1}, \cdots, l_{n}\right)^{\prime}, 1=(1, \cdots, 1)^{\prime}, \mathrm{A}_{n \times K}=\left(a_{1}, \cdots, a_{n}\right)^{\prime}$ and $\Phi_{K \times K}=\left(\varphi_{j k}\right)=\left(\int_{T} \phi_{j}(t) \phi_{k}(t) \mathrm{d} t\right), \quad j, k=1, \cdots, K$.

The main problem is to approximate the basis coefficients of sample curves from their discrete observations and to select an appropriate basis by taking into account the characteristics of the observed sample curves. Due to the high dependence structure of the design matrix A $\Phi$ in the model, the multiple linear regression model shows high multicollinearity. Functional PCA and functional PLS approaches as described in Section 2.3.2 were used here so as to construct a reduced set of uncorrelated components of the design matrix. The problem is then reduced to regressing a logit transformation of response variable on an optimum set of orthogonal (principal or PLS) components obtained as generalized linear combinations of the functional predictor that solve optimization criteria 
in Equations (2) and (3). In the rest part of this paper, FPCA and FPLS are used to refer to functional PCA logistic regression model and functional PLS logistic regression model, respectively.

The FPCA and FPLS were carried out with $k$, the number of component basis functions, ranging from 2 to 30 and $k$ was optimized by using the leave-out-onesite cross-validation as mentioned in Section 2.3.1.

All the algorithms for computations and analyses were implemented in R statistical programming language [25] using the R packages MASS and fda.usc [26].

\section{Results and Discussion}

The mean spectral patterns from hyperplastic and adenomatous polyps after standard pre-treatment are shown in Figure 1. It illustrates that the spectra of the two types of polyps are of very similar overall shape, with the mean spectra showing small between-class differences.

\subsection{Discrimination by PCDA and PLSDA Models}

For PCDA and PLSDA models, the discrimination results of cross-validation were used to optimize the number of PCs or PLS components. In Table 1, the PCDA model constructed on the first twenty PCs gave the best discrimination accuracy with sensitivity and specificity of $81 \%$ and $76 \%$, respectively. When

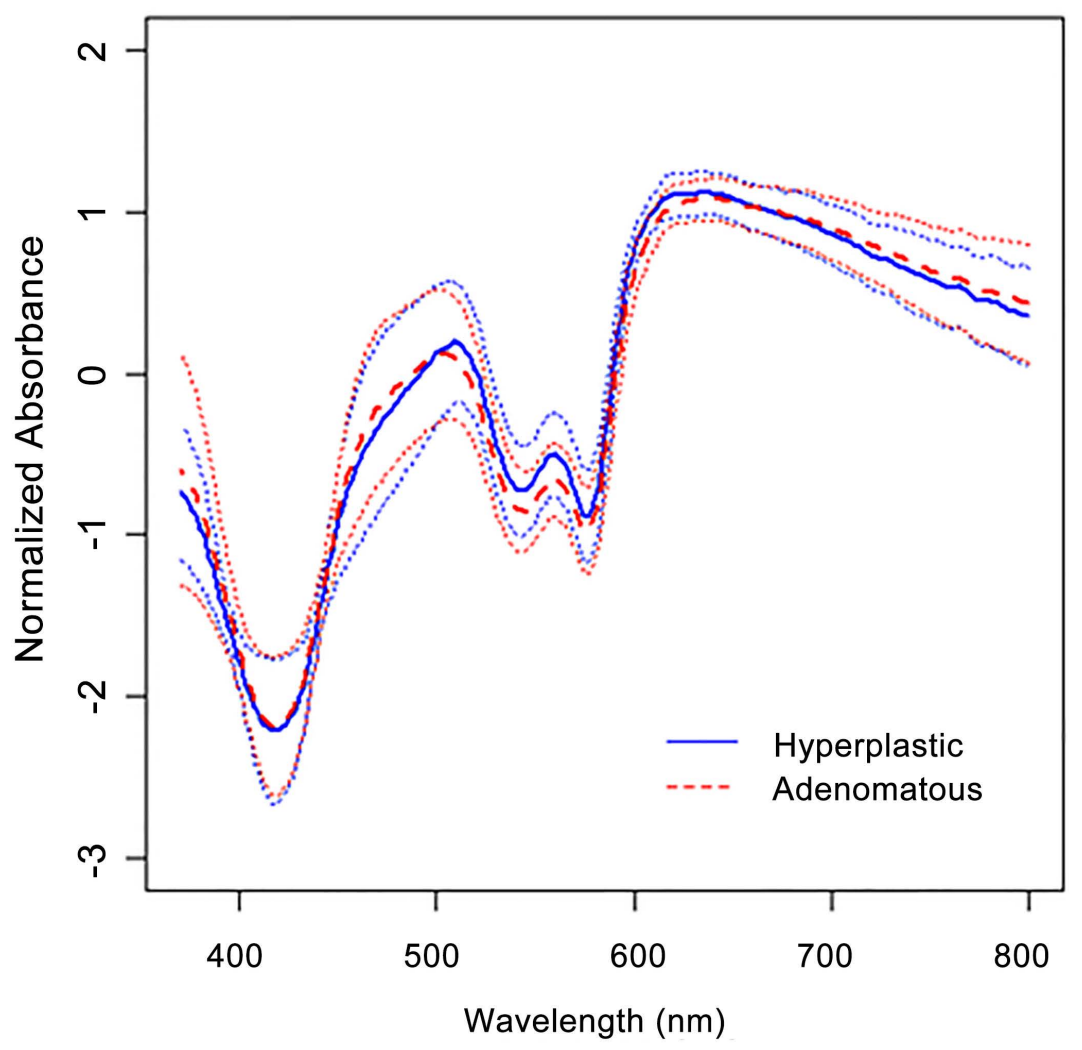

Figure 1. Mean spectra ( \pm one standard deviation) from hyperplastic (solid blue line for mean and dotted blue line for standard deviation) and adenomatous (dashed red line and dotted red lines for standard deviation) polyps of colon data after standard pre-treatment. 
taking into account the relationship between the spectral variables and the response variable for latent variable design, the optimal PLSDA model achieved a leave-one-out cross-validation discrimination accuracy with the same sensitivity and specificity of $81 \%$ and $76 \%$ respectively, but using only seven PLS components in constructing the canonical variable.

By combining the loadings from the PCA or PLS and LDA, the PCDA loading and PLSDA loading can be found to show the contribution at each wavelength to the linear discrimination rule and thus can be related easily to the spectral features for interpretation purpose.

However, in Figure 2 the loadings from these two models look complex, especially for the PCDA model with more number of components used for the discrimination, thus the contribution of each wavelength to the classification becomes less interpretable when it is related to the spectral features.

\subsection{Discrimination by FPCA and FPLS Logistic Regression Models}

Though the PLSDA model used a smaller number of constructed variables for classification when compared with the PCDA model, the classification performance and interpretation of both models are not satisfactory. FPCA and FPLS logistic regression models showed improvements over the multivariate methods.

Table 1. Classification results of different models using leave-one-out cross-validation for differentiating between hyperplastic and adenomatous polyps of colon data.

\begin{tabular}{cccc}
\hline Models & Parameter & Sensitivity & Specificity \\
\hline PCDA & $k=20$ & $81 \%$ & $76 \%$ \\
PLSDA & $k=7$ & $81 \%$ & $76 \%$ \\
FPCA & $k=12$ & $88 \%$ & $76 \%$ \\
FPLS & $k=7$ & $85 \%$ & $76 \%$ \\
\hline
\end{tabular}
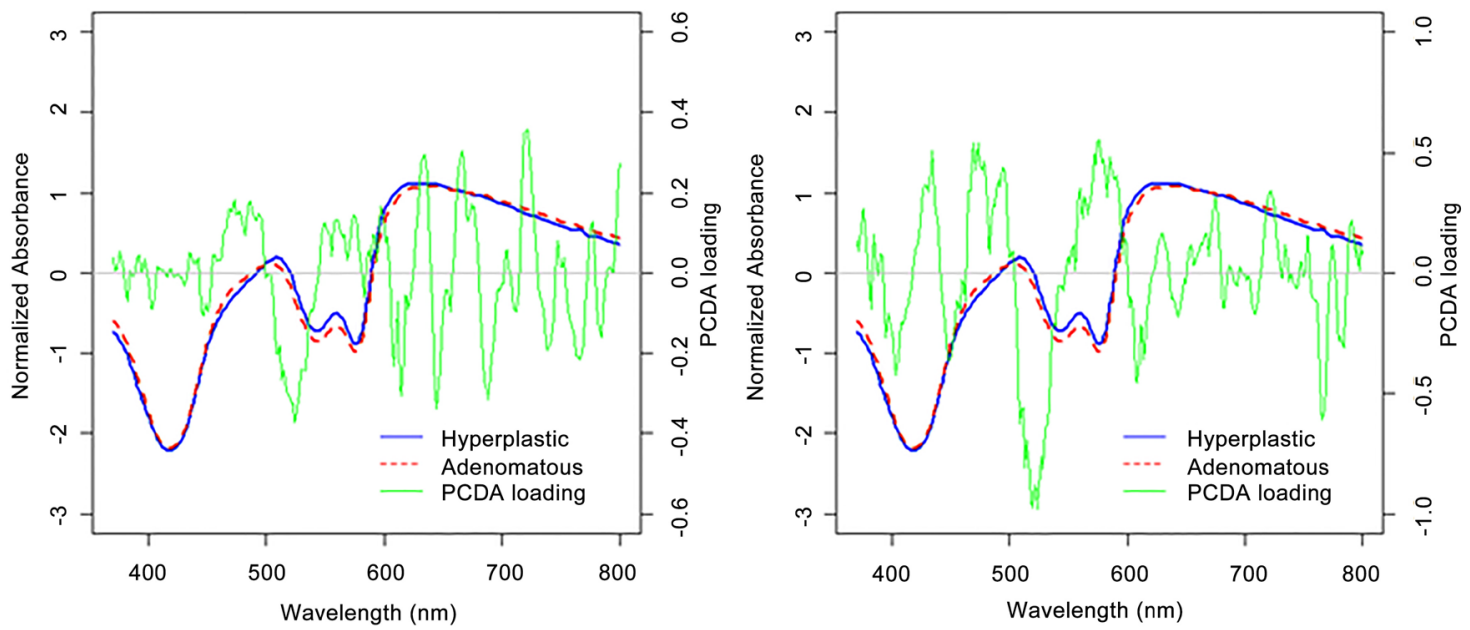

Figure 2. PCDA loadings (left panel) and PLSDA loadings (right panel) for discrimination between hyperplastic and adenomatous polyps of colon data. The discrimination loading is shown in green, with the mean spectra for the two types superimposed (hyperplastic solid blue line, adenomatous dashed red line). 
The discrimination results of cross-validation were used to optimize the number of PCs or PLS components basis functions. In Table 1, the FPCA models with twelve functional PCs gave the optimal results, with sensitivity and specificity of $88 \%$ and $76 \%$ respectively. The FPLS models with seven functional PLS components gave the optimal results, with both sensitivity and specificity of $85 \%$ and $76 \%$ respectively. Here a specificity of $76 \%$ was chosen considering the small size of colon data so as to make sensitivity comparable for different models. As shown in Figure 3, when we compare the FPCA models with the PCDA models having the same number of components, for the models using more than ten PCs, the FPCA model can often achieve higher classification accuracy than PCDA model. When we compare the FPLS models with the PLSDA models having the same number of components, for the models using at least seven PLS components, the FPLS model can often achieve higher classification accuracy than PLSDA model.

Using a small number of PC or PLS functional basis components, the coefficients of FPCA and FPLS models show simpler structure features. This allows the visualization of discrimination coefficient vectors and showed how they contribute to the correct classification clearly. In Figure 4, the obvious features with large positive loadings from both FPCA and FPLS models concentrated at around $410 \mathrm{~nm}, 540 \mathrm{~nm}$ and $580 \mathrm{~nm}$, corresponding to some small differences between the mean spectra of hyperplastic and adenomatous polyps. The effect of absorption seems to play a key role in the spectral differences and the classification between two types of tissue in some specific spectral region. These informative regions might be due to absorption dips of $\mathrm{HbO} 2$ at $415 \mathrm{~nm}, 542 \mathrm{~nm}$ and $577 \mathrm{~nm}$ in the spectra of high risk cancer due to increased $\mathrm{Hb}$ concentration [27]. It is known that cancers and pre-cancerous tissues are characterized by increased microvascular volume, hence increased blood content [28].
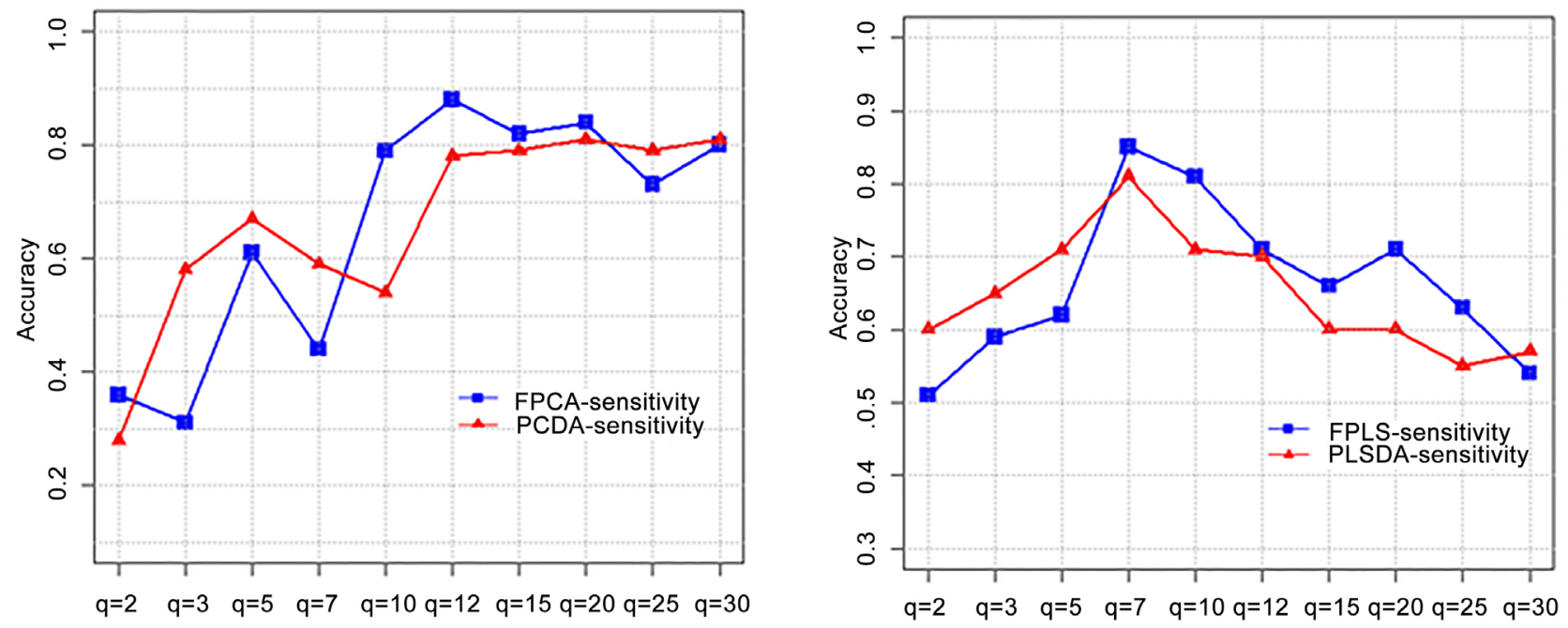

Figure 3. Left panel: FPCA and PCDA classification accuracy of colon data as measured by sensitivity; right panel: FPLS and PLSDA classification accuracy of colon data as measured by sensitivity. 
We further explored the structures of the FPCA and FPLS components. The first four functional PCs were considered since they account for more than 95\% of the total variation in the colon spectral data. As shown in Figure 5, the first and dominant component is simple in structure and resembles a single cycle of a sine wave. The first two functional PCs with large positive or negative loadings around $410 \mathrm{~nm}, 540 \mathrm{~nm}$ and $580 \mathrm{~nm}$ explain the informative spectral features in these regions. The subdominant components are roughly sinusoidal, but show
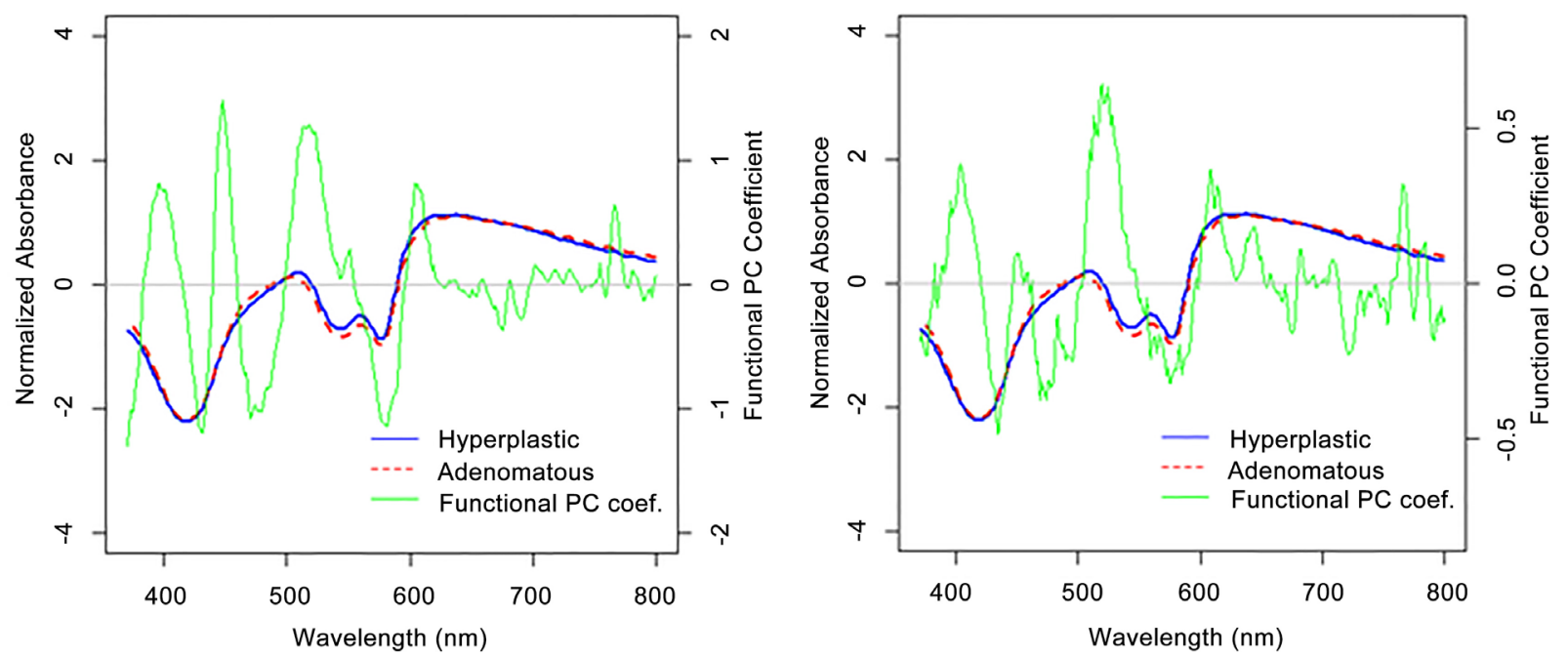

Figure 4. FPCA loadings (left panel) and FPLS loadings (right panel) for discrimination between hyperplastic and adenomatous polyps of colon data. The functional regression loading is shown in green, with the mean spectra for the two types superimposed (hyperplastic solid blue line, adenomatous dashed red line).
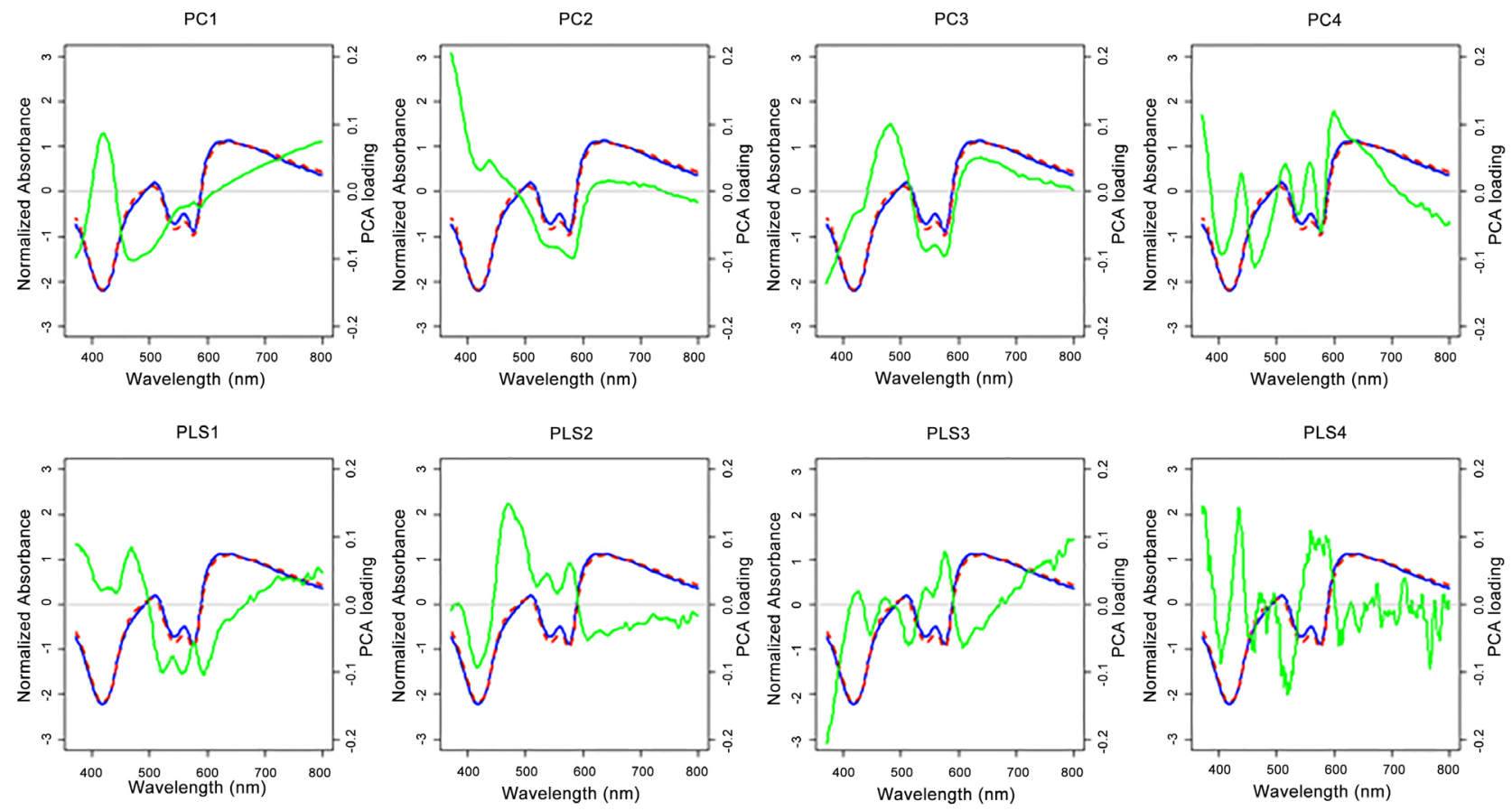

Figure 5. The first four functional PC loadings (top panel) and the first four functional PLS loadings (bottom panel) from colon data. Functional PC loading and functional PLS loading are shown in green line, with the mean spectra for the two types of polyps superimposed (hyperplastic in solid blue line, adenomatous in dashed red line). 
more and more cycles. The functional PLS loadings seem to capture more informative spectral features even for dominant component.

\section{Conclusions}

Functional data analysis has been widely used in spectroscopic data where the absorbance spectrum is a functional variable whose observations are functions of wavelength. In this study, two functional logistic regression models with functional PC basis and functional PLS basis have been developed to distinguish adenomatic polyps from hyperplastic polyps during endoscopy of the colon. The results of this study showed that the functional logistic regression models outperformed the PCDA and PLSDA models by using a small number of components.

The commonly used multivariate models PCDA and PLSDA with more discriminant components used in the models may include some noise to hinder the classification performance and the interpretation of the spectral feature may be challenging. Taking into account the functional form of spectroscopic data, the FPCA and FPLS logistic regression models improved classification accuracy. The functional representation of the spectra combines dimension reduction and smoothing in one step. Both the FPCA and FPLS models gave better classification performance than the PCDA and PLSDA models and used a reduced number of functional basis components. In particular, FPLS used fewer latent variables than FPCA.

The most important contribution of the FPCA and FPLS models is that the discrimination coefficients that contributed to the correct classification of hyperplastic and adenomatic polyps provided us insights and good understanding of the complex spectral features related to different types of colon polyps. With substantial reduction of spectral components, this functional logistic regression model is also a potentially accurate, fast and robust tool to distinguish adenomatic polyps from hyperplastic polyps during endoscopy of colon for the detection and removal of precancerous polyps before they turn into cancer. This is crucial for real-time clinical diagnostic application. In the future it is of interest to test the models on a larger number of colonic polys samples to give a more reliable diagnostic result. The spectral feature selection in functional data classification and interpretation of the classification loadings will also be further developed in future work.

\section{Acknowledgements}

This research is funded by Academic Research Funds (AcRF: RI 6/14 ZY) of National Institute of Education, Nanyang Technological University, Singapore. The author thanks the National Medical Laser Centre of University College London for providing the vis-NIR spectroscopic data.

\section{References}

[1] Saeys, W., de Ketelaere, B. and Darius, P. (2008) Potential Applications of Func- 
tional Data Analysis in Chemometrics. Journal of Chemometrics, 22, 335-344. https://doi.org/10.1002/cem.1129

[2] Chalmers, J.M. and Griffiths, P.R., Eds. (2002) Handbook of Vibrational Spectroscopy. John Wiley \& Sons Ltd., Chichester.

[3] Johnson, R.A. and Wichern, D.W. (1992) Applied Multivariate Statistical Analysis. 3rd Edition, Prentice Hall, Upper Saddle River.

[4] Næs, T. and Martens, H. (1998) Principal Component Regression in NIR Analysis: Viewpoints, Background Details and Selection of Components. Journal of Chemometrics, 2, 155-167. https://doi.org/10.1002/cem.1180020207

[5] Geladi, P. and Kowalski, B.R. (1986) Partial Least-Squares Regression: A Tutorial. Analytica Chimica Acta, 185, 1-17.

[6] Wold, S. (2001) Personal Memories of the Early PLS Development. Chemometrics and Intelligent Laboratory Systems, 58, 83-84.

[7] Ramsay, J. and Silverman, B. (1997) Functional Data Analysis. Springer, New York. https://doi.org/10.1007/978-1-4757-7107-7

[8] Aguilera, A.M., Escabias, M., Preda, C. and Saporta, G. (2010) Using Basis Expansions for Estimating Functional PLS Regression: Applications with Chemometric Data. Chemometrics and Intelligent Laboratory Systems, 104, 289-305.

[9] Zhao,Y., Ogden, R.T. and Reiss, P.T. (2012) Wavelet-Based Lasso in Functional Linear Regression. Journal of Computational and Graphical Statistics, 21, 600-617. https://doi.org/10.1080/10618600.2012.679241

[10] Pourshoghi, A., Zakeri, I. and Pourrezaei, K. (2016) Application of Functional Data Analysis in Classification and Clustering of Functional Near-Infrared Spectroscopy Signal in Response to Noxious Stimuli. Journal of Biomedical Optics, 21, Article ID: 101411. https://doi.org/10.1117/1.JBO.21.10.101411

[11] James, G.M. (2002) Generalized Linear Models with Functional Predictors. Journal of the Royal Statistical Society: Series B (Statistical Methodology), 64, 411-432. https://doi.org/10.1111/1467-9868.00342

[12] Müller, H. and Stadtmüller, U. (2005) Generalized Functional Linear Models. The Annals of Statistics, 33, 774-805. https://doi.org/10.1214/009053604000001156

[13] Reiss, P.T. and Ogden, R.T. (2007) Functional Principal Component Regression and Functional Partial Least Squares. Journal of the American Statistical Association, 102, 984-996. https://doi.org/10.1198/016214507000000527

[14] Ratcliffe, S.J., Heller, G.Z. and Leader, L.R. (2002) Functional Data Analysis with Application to Periodically Stimulated Foetal Heart Rate Data. II: Functional Logistic Regression. Statistics in Medicine, 21, 1115-1127.

https://doi.org/10.1002/sim.1068

[15] Savitzky, A. and Golay, M.J.E. (1964) Smoothing and Differentiation of Data by Simplified Least-Squares Procedures. Analytical Chemistry, 36, 1627-1639. https://doi.org/10.1021/ac60214a047

[16] Barnes, R.J., Dhanoa, M.S. and Lister, S.J. (1989) Standard Normal Variate Transformation and De-Trending of Near-Infrared Diffuse Reflectance Spectra. Applied Spectroscopy, 43, 772-777. https://doi.org/10.1366/0003702894202201

[17] Davies, A.M.C. and Fearn, T. (2005) Back to Basics: The Principles of Principal Component Analysis. Spectroscopy Europe, 16, 20-23.

[18] Naes, T., Isaksson, T., Fearn, T. and Davies, T. (2002) A User-Friendly Guide to Multivariate Calibration and Classification. NIR Publications, Chichester.

[19] Barker, M. and Rayens, W. (2003) Partial Least Squares for Discrimination. Journal 
of Chemometrics, 17, 166-173. https://doi.org/10.1002/cem.785

[20] Roger, J.M., Palagos, B., Guillaume, S. and Bellon-Maurel, V. (2005) Discriminating from Highly Multivariate Data by Focal EIGEN Function Discriminant Analysis; Application to NIR Spectra. Chemometrics and Intelligent Laboratory Systems, 79, 31-41.

[21] Zhu, Y. and Tan, T.L. (2016) Penalized Discrimination Analysis for the Detection of Wild Grown and Cultivated Ganoderma lucidum Using Fourier Transform Infrared Spectroscopy. Spectrochimica Acta Part A: Molecular and Biomolecular Spectroscopy, 159, 68-77.

[22] Zhu, Y. and Tan, T.L. (2015) Discrimination of Wild-Grown and Cultivated Ganoderma lucidum by Fourier Transform Infrared Spectroscopy and Chemometric Methods. American Journal of Analytical Chemistry, 6, 480-491.

https://doi.org/10.4236/ajac.2015.65047

[23] Zhu, Y. and Tan, T.L. (2015) Chemometric Feature Selection and Classification of Ganoderma lucidum Spores and Fruiting Body Using ATR-FTIR Spectroscopy. American Journal of Analytical Chemistry, 6, 830-840. https://doi.org/10.4236/ajac.2015.610079

[24] Preda, C., Saporta, G. and Leveder, C. (2007) PLS Classification for Functional Data. Computational Statistics, 22, 223-235. https://doi.org/10.1007/s00180-007-0041-4

[25] R Core Team (2012) R: A Language and Environment for Statistical Computing. R Foundation for Statistical Computing, Vienna.

[26] Febrero-Bande, M. and Oviedo de la Fuente, M. (2012) Statistical Computing in Functional Data Analysis: The R Package fda.usc. Journal of Statistical Software, 51, 1-28. https://doi.org/10.18637/jss.v051.i04

[27] Zijlstra, W.G., Buursma, A. and Assendelft, O.W. (2000) Visible and Near Infrared Absorption Spectra of Human and Animal Haemoglobin: Determination and Application. VSP International Science Publishers, Netherlands.

[28] Jain, R.K. (1988) Determinants of Tumor Blood Flow: A Review. Cancer Research, 48, 2641-2658.

Submit or recommend next manuscript to SCIRP and we will provide best service for you:

Accepting pre-submission inquiries through Email, Facebook, LinkedIn, Twitter, etc. A wide selection of journals (inclusive of 9 subjects, more than 200 journals)

Providing 24-hour high-quality service

User-friendly online submission system

Fair and swift peer-review system

Efficient typesetting and proofreading procedure

Display of the result of downloads and visits, as well as the number of cited articles

Maximum dissemination of your research work

Submit your manuscript at: http://papersubmission.scirp.org/

Or contact ajac@scirp.org 